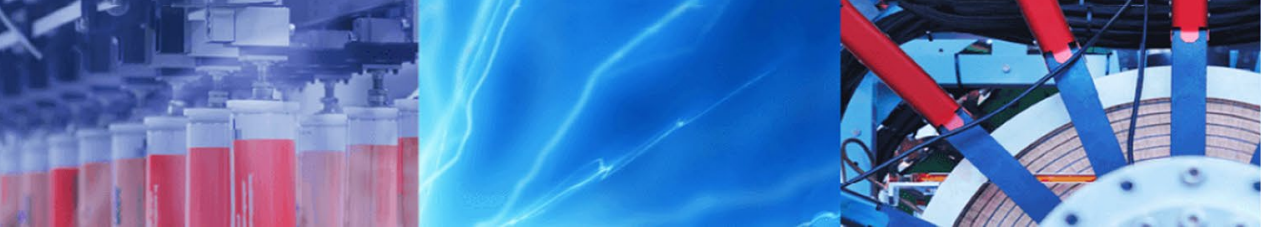

Research Article

\title{
A hybrid energy-efficient routing protocol for wireless body area networks using ultra-low-power transceivers for eHealth care systems
}

\author{
Ch. Rajendra Prasad ${ }^{1,2}$ (Dolaiah Bojja ${ }^{1}$
}

Received: 16 April 2020 / Accepted: 18 November 2020 / Published online: 30 November 2020

(c) Springer Nature Switzerland AG 2020

\begin{abstract}
One of the major applications of Wireless Body Area Networks (WBANs), and the Internet of Things is the Electronic Health-Care systems. The progress in WBANs, and implanted health monitoring technologies have strong potential to alter the future of healthcare services by enabling ubiquitous monitoring of patients. An energy-efficient protocol is an important factor in the operation of eHealth care systems. A hybrid energy-efficient routing protocol is proposed in this paper for the distributed wireless body area network. In which a hybrid communication method and a new synchronization scheme are discussed. The hybrid communication enhances the network lifetime, and the synchronization scheme will consume less energy by avoiding collisions. The performance of the proposed protocol is analyzed, and it is compared with three baseline routing protocols at the $2.4 \mathrm{GHz}$ ISM band with two ultra-low power transceivers.
\end{abstract}

Keywords eHealth care $\cdot$ Internet of Things $\cdot$ WBANs $\cdot$ REAST $\cdot$ Routing protocols $\cdot$ Ultra-low-power radio transceiver

\section{Introduction}

Recent advances in Wireless Body Area Networks (WBANs) and ubiquitous computing have revealed the widespread applications of these techniques in the medical field, particularly related to Cardio Vascular Disease (CVD), stroke, hypertension, and diabetes treatment. The deployment of wireless sensors, in this context, lies in the human body, fostering the emergence of a new notion in this field. The WBAN brings a myriad of possibilities for human health remote monitoring for the enhancement of a patient's quality of life. According to the World Health Organization (WHO), by $2030,31 \%$ of worldwide deaths occur due to CVD and diabetes [1]. In 2015, the WHO clearly stated that, if the diseases are not identified in the early stages, the medicare expenditures for chronic diseases could increase drastically [2]. However, the advancements in the Internet of Things (IOT) in conjunction with the Wireless Body
Area Network (WBAN) are used for prior identification and prophylaxis of possible chronic conditions. The health conditions or parameters of chronic diseases are continuously monitored by embedding Ultra Low Power (ULP) wearable and/or implanted sensors in WBAN systems. The physiological parameters, like electroencephalography (EEG), electrocardiogram (ECG), body temperature, respiratory rate, and movement, etc, are recorded by the sensors within a WBAN, and these recorded data are transmitted wirelessly to sink for further processing and examination. The advanced medicare system keeps the track of chronically ill and aged patients from their living places, where they are most comfortable and relaxed, which in turn reduces Medicare expenses and often minimizes regular health checkups.

Figure 1 illustrates the four-layer eHealth-Care system's architecture [3]. Layer I incorporate several sensor nodes that operate within a WBAN. These nodes

Ch. Rajendra Prasad, chrprasad20@gmail.com; rajendra_prasad_ch@srecwarangal.ac.in; Polaiah Bojja, polaiah@kluniversity.in | 'Department of ECE, Koneru Lakshmaiah Education Foundation (KLEF), Green Fields, Vaddeswaram, Andhra Pradesh 522502, India. ${ }^{2}$ Department of ECE, School of Engineering, SR University, Warangal, Telangana 506371, India. 


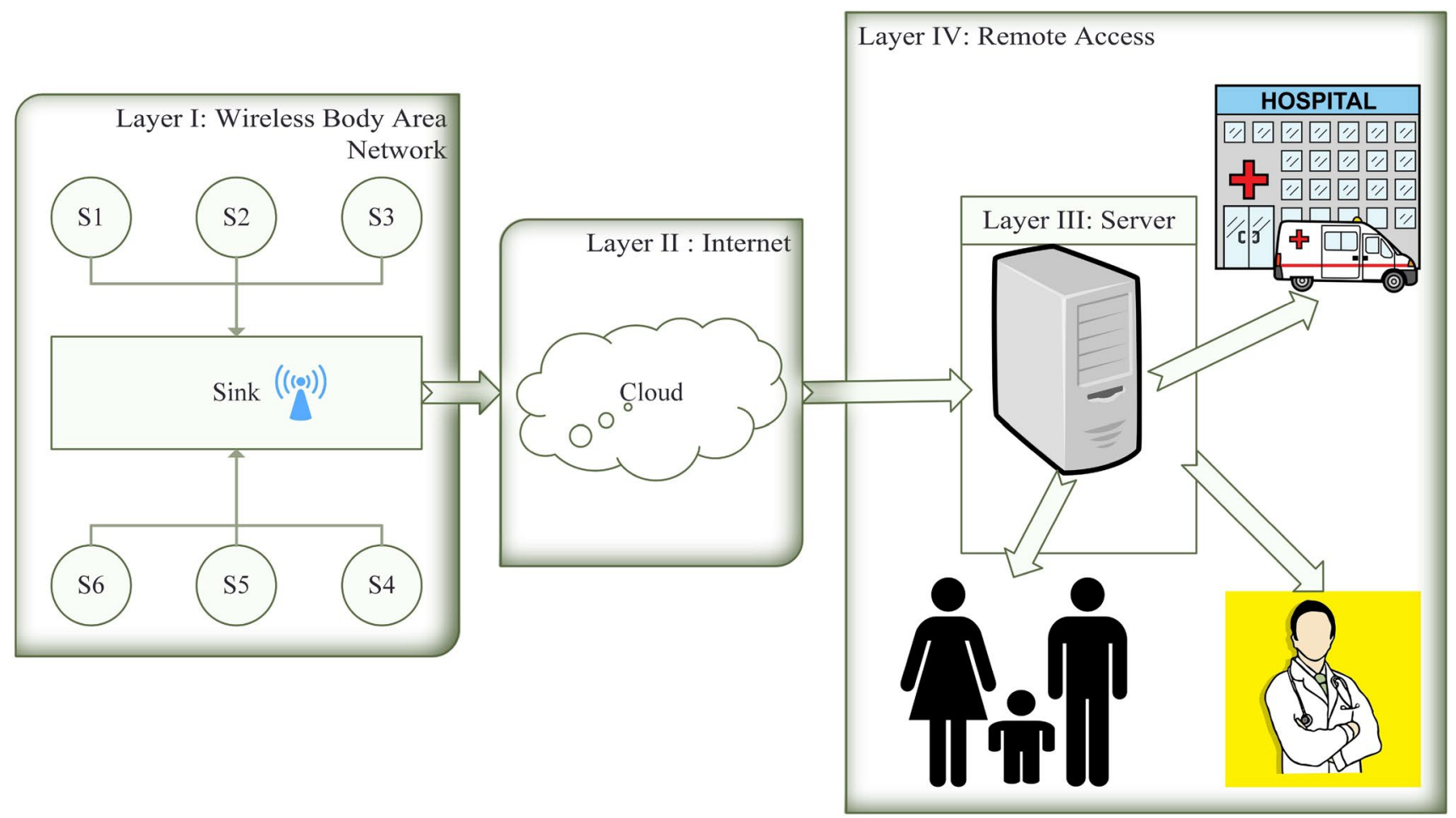

Fig. 1 Four layer eHealth care system architecture

are deployed on the patient's body as wearable and/ or implanted sensor nodes. All these sensors gather the patient's vital information and transmit to the sink node, which captures, aggregates the information, and transmits to layer II.

Layer II acts as a transmission medium, and further, it transmits the information to the central server in layer III through the internet. In layer III, the information received is filtered, analyzed, and decisions are taken based on the raw information. This processed data is further conveyed to Layer IV. layer IV offers Medicare facilities to the patients. The examined data is available in the server and accessible to the Doctors, eHealth-care employees, and family members remotely. Layer IV provides two different services viz., emergency and healthcare services.

The main contributions of this work are as follows:

- A priority-based route is chosen to transfer data from a set of available paths from a sensor node to the sink to provide optimum energy consumption in the entire network.

- If the temperature of the node exceeds critical temperature, avoid the route to minimize radiation effects on patient sensitive organs.

- Hybrid synchronization with variable time slot assignment for the reliable data transmission from every sensor node towards the sink node.
This paper is organized in such a way that the related work is described in Sect. 2. The implementation of the proposed hybrid energy-efficient protocol is detailed in Sect. 3. The simulation results are presented in Sect. 4. The discussion of the proposed method with traditional methods is presented in Sect. 5. Eventually, conclusions are provided in Sect. 6 .

\section{Related work}

An intelligent healthcare system with a target-specific flexible quality of service (QoS) consisting of an intelligent gateway, wireless communication links, and a set of sensor nodes is to periodically monitor and communicate human essential signals to a distant healthcare server. The eHealth-care model remotely monitors the health status of the patients and accessible for physicians and nurses [4]. Besides, a large amount of data gathered enables researchers to develop advanced medicare devices. WBAN is an advanced wireless topology, which provides the possibility of early identification of unusual health conditions, real-time tracking of medicare, and remote e-medicine services for individuals [5].

WBAN's characteristics vary based on the transmission energy limitations, propagation medium, and human organ/tissue security requirements from traditional 
wireless communication systems [6]. For enhancing the quality of human life, health, loT is one of the most promising methods. This is achieved by monitoring healthcare, and remote telemedicine support systems, which enable data collection, communication, and visualization through the internet in real-time $[7,8]$. The first global standard for wireless transmission is the IEEE 802.15.6 standard that optimizes energy usage and offers security for healthcare, non-healthcare systems in or around the patient body [9]. The idea of telemedicine under the health loT has shifted from remote medicine to personalized universal healthcare systems. Concerning to home or hospital medicare surveillance context, the wireless implanted medicare surveillance equipment considerably enhances patient convenience and mobility in contrast to wired healthcare systems. Radio Frequency can cover enhanced operating distances and allow wireless communication with on-body devices by interactive implanted sensors and devices [10].

Besides, the energy efficiency of the Radio Frequency modules is the main aspect of the in-to-out body channel owing to the practical limitations of batteries. These limitations are minimized by employing miniaturized embedded transceivers in WBANs [11]. The wireless routing protocols are established for the discovery, and testing of the most energy-efficient path. The relay node approach leads to the minimized energy utilization of the implanted sensor nodes by selecting the shortest route. A two-relay node approach trade-off with reliable transmission and energy efficiency is contrasted to the single relay approach of direct transmission. Two-way communication technique mainly focuses on wireless fading channels to get significant attention to improve spatial diversity, and energy efficiency [12].

In [13] authors presented an energy-aware routing (EAR) protocol that is employed for Medicare systems. A change in network topology results in the rapid depletion of battery energy, which results in an overall reduction in network efficiency. Homogeneous transmission leads to more overhead on distant nodes and heterogeneous communication rapidly depletes the battery energy of adjoining nodes. The authors in [14] use the homogeneous transmission to send information directly to the personal server from the biosensor nodes. This approach beats the delay in transmission with the cost of more energy consumption for the distant biosensor nodes. By concentrating on these factors, a route is selected for data transmission.

In [15], the authors demonstrated a tree-structured priority-oriented protocol for WBANs. They used specific paths for critical data delivery and if it is successful, then normal data is put forward for transmission. Due to dedicated channels in this topology, frequent loss of accessible resources is possible. In [16], the authors proposed adaptive route allocation based on the remaining energy at the bio-sensor node. This dynamic route allocation assures equal load distribution on the nodes and extends the lifetime of the network. In [17], the authors presented routing protocol design constraints for WBANs. The foremost constraint is the number of forwarder nodes and a later constraint is the deployment of a forwarder node in the topology of WBANs. As the number of forwarders is increased, the overall network setup expenditure also increases, and hence, the cost is another major constraint on the network topology.

This paper presented a hybrid routing protocol, which employs ULP radio transceivers, hybrid transmission, and novel synchronization. The hybrid nature of transmission between the sensor nodes and the sink increases the lifetime of the network. The synchronization scheme reduces energy consumption by preventing collisions in data transmission. The radio transceivers employed in this paper are ULP transceivers such as Nordic nRF2401A, NRF24L01 at $2.4 \mathrm{GHz}$ ISM band. These transceivers are having optimized energy in terms of supply voltage, transmitter, and receiver and amplifier powers. The utilization of ULP transceivers further minimizes the energy consumption of the entire WBAN.

\section{Proposed model for WBANs}

The proposed hybrid protocol addresses two main problems faced by WBANs with conventional protocols such as the lifetime of the network and energy consumption. The lifetime of the entire network is improved by employing hybrid communication between the sensor nodes and the sink. The energy utilization is minimized by employing a hybrid synchronization technique. Furthermore, energy expenditure is minimized by using Nordic nRF2401A, NRF24L01 at $2.4 \mathrm{GHz}$ ISM band. The complete description of this protocol is discussed in the next subsections.

\subsection{Network deployment}

In this protocol, one sink and eight different sensor nodes are deployed according to the parameters mentioned in Table 1. The placement of sensor nodes on the patient body and communication among the biosensor nodes is illustrated in Fig. 2.

\subsection{Hybrid communication}

The communication between the nodes and the sink is either direct or indirect as shown in Fig. 2. Direct communication leads to more energy consumption and indirect communication leads to delay in data delivery. We implemented hybrid communication i.e, direct and indirect 
Table 1 Deployment area, the position of the sink and the sensor nodes

\begin{tabular}{ll}
\hline Parameter & Value \\
\hline Area of deployment & $0.8 \times 1.8 \mathrm{~m}^{2}$ \\
Sensors & 8 \\
Sink position & $0.4(\mathrm{x}), 0.9(\mathrm{y})$ \\
Sensors placement & $1(0.6,1.05), 2(0.75,0.6), 3(0.5,0.75), 4(0.5$, \\
& $0.4), 5(0.3,0.1), 6(0.35,0.6), 7(0.35,0.95)$, \\
& $8(0.4,1.6)$ \\
Initial energy & 0.4 Joules \\
Path loss coefficient & 4.1 \\
\hline
\end{tabular}

transfer of data between the sink and the sensor nodes to enhance the lifetime of the entire network. Hybrid communication is a combination of direct and indirect data transfer, which optimizes the energy of the network and it will improve the lifetime of the network. Hybrid communication addresses two issues such as energy consumption, and temperature effects on patient body tissues.

\subsubsection{Energy consumption model}

After the initialization of the network, a priority-based route is chosen for the transfer of data from a set of available paths from the sensor node to the sink. Sensor nodes with emergency information are given with the highest priority, which can follow direct transmission to the sink. Let us consider the total number of sensor nodes is $N, S$ is the sink, $\zeta$ is the relay node, which transmits the data between the sink and the node, and $N_{C}$ is the network capacity. The $D$ is the entire data that is routed through the relay node. According to the deployment of sensor nodes, link connectivity parameters are defined in Eqs. (1) and (2).

$L_{i \zeta}= \begin{cases}1, & \text { i data routed via } \zeta \\ 0, & \text { otherwise }\end{cases}$

$L_{i S}= \begin{cases}1, & i \text { routed directly to the } S \\ 0, & \text { otherwise }\end{cases}$

$L_{i \zeta}$ is the routing element between $i$ and $\zeta . L_{i s}$ is routing element between $i$ and $S . D_{t s}$ is the total data transmitted

\section{Network deployment and communication between sink and the sensor nodes}

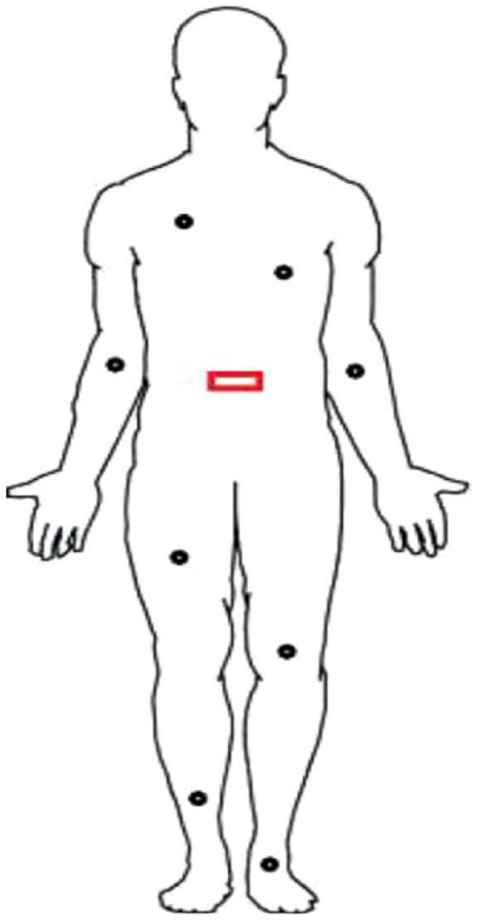

Network Deployment

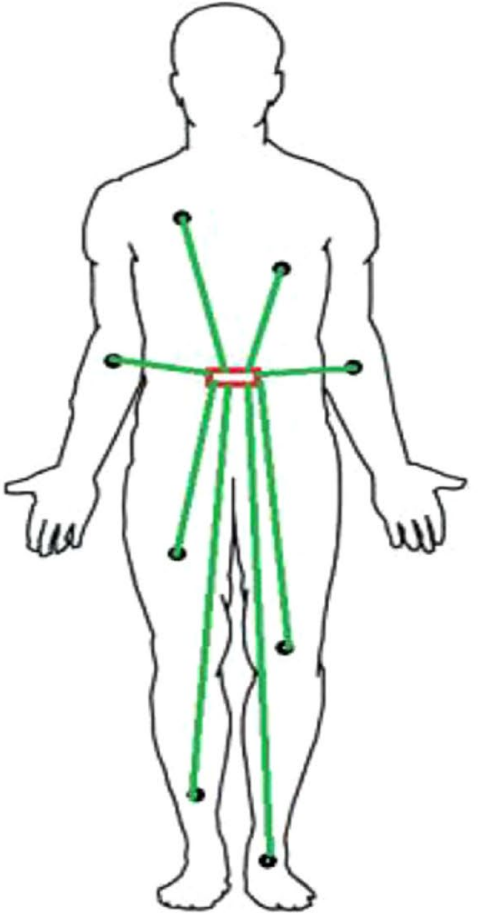

Direct Communication

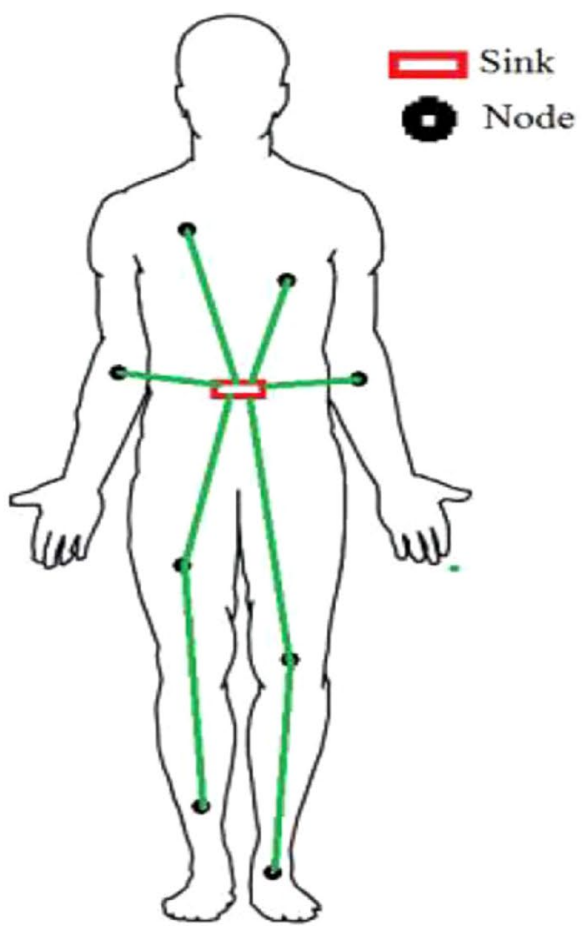

Multi-Hop Communication

Fig. 2 Network deployment and communication between the sink and sensor nodes 
from the relay node $(\zeta)$ to the sink $(S) . D_{i \zeta}$ is the data flow from the individual sensor (i) to the relay node ( $\zeta$ ). Equation (3) represent an optimized energy dissipation model, which is the sum of energy consumed by relay node $\left(E_{\zeta}\right)$ and sensor nodes.

$\min \sum_{i \in N} E_{t}=\sum_{i \in N}\left(E_{t}+E_{\zeta}\right)$

where:

$$
\begin{aligned}
\sum_{i \in N} E_{i T_{x}} & =\sum_{i \in N} L_{i \zeta} d_{i \zeta}\left(n \times E_{a m p} \times D^{n}+E_{T-\text { elec }}\right) \\
\sum_{i \in N} E_{i R_{x}} & =\sum_{f \in N} L_{i \zeta} d_{i \zeta}\left(E_{R-\text { elec }}\right) \\
\sum_{\zeta \in N} E_{\zeta T_{x}} & =\sum_{\zeta \in N} \zeta_{s}^{t}\left(E_{a m p} \times n \times D^{n} \times E_{d a}+E_{T-\text { elec }}\right) \\
\sum_{\zeta \in N} E_{\zeta R_{x}} & =\sum_{\zeta \in N} L_{i \zeta} d_{i \zeta}\left(E_{T-\text { elec }}\right)
\end{aligned}
$$

The total energy expenditure is the addition of the energy of direct $\left(E_{\text {direct }}\right)$ and multihop $\left(E_{M-h o p}\right)$ communications and is given as:

$E_{\text {overall }}=E_{\text {direct }}+E_{M-H o p}$

$E_{\text {direct }}=E_{t x}=E_{r x}=d^{2}\left(E_{\text {ele }}+E_{\text {amp }}\right) b$

$E_{M-H o p}=n b\left(E_{t x}\right)+b(n-1)\left(E_{d a}+E_{r x}\right)$

\subsubsection{Temperature effects}

The deployed sensor node's temperature is increased as they continuously transmit the data, which leads to damage to the patient's sensitive organs [18, 19]. The abovespecified problem can be addressed by diverting the transmission to another sensor node by estimating the temperature at every node. The damage of patient-sensitive organs due to temperature effects is minimized by employing Eq. (7). Where $\operatorname{Tmp}(i)$ is the temperature at the $i^{\text {th }}$ sensor node. Based on Tem(i), this protocol establishes the link $L(i)$ if $\operatorname{Tmp}(i)$ is less than the critical temperature $\left(C_{i}\right)$, otherwise, the link $L(i)$ is not established at the $i^{\text {th }}$ node.

$L(i)= \begin{cases}1, & C_{i}<\operatorname{Tmp}(i) \\ 0 & C_{i} \geq \operatorname{Tmp}(i)\end{cases}$

\subsection{Hybrid synchronization}

The hybrid communication utilizes the optimized energy, which enhances the network lifetime. However, the actual energy consumption problem is addressed by a hybrid synchronization method. A scheduling scheme with differentiated traffic for WBANs has also been proposed [20]. TDMA method itself costs extra delay for sporadic synchronization [21]. For $N$ number of rounds, synchronization of all sensor nodes further increases the delay, which in turn reduces the stability of the network and deplete more energy of the network. To address the delay, this protocol primarily allocates time slots (TSs) according to the basic TDMA method. From the next round on words, it assigns variable TSs based on the previous arrival information. The variable TSs are computed based on the previous delay and the drift value given in Eqs. (8) and (9).

$D=\min \left(T S_{1}, T S_{2}, \ldots T S_{n}\right) \times \frac{F}{100}$

$D V=\left\{\begin{array}{lc}\nabla T, & |\nabla T|>D \\ 0, & \text { otherwise }\end{array}\right.$

where: $\nabla T=$ Expected arrival time - Present arrival time

Based on a deployment model of the sensor nodes, the sink assigns TSs and sends a request to replay TSs. Depending on the sensor node requirements, the sink assigns variable TSs for the sensor nodes. Every TS will consist of information packet, acknowledgment, and suitable delay depends on deployment topology. A Guard band Time $\left(T_{G B}\right)$ is inserted between the successive TSs to provide variable synchronization, as illustrated in Fig. 3.

The allocation of TSs with $T_{G B}$ value varies on the period of the successive TSs and is estimated by using Eqs. (10), (11) and (12). The probable interface and collision clock drift are prevented by using an adaptive guard band.

$T_{n, n+1}^{G B}=\frac{F}{200} \times\left[T S_{n}+T S_{n+1}\right]$

$T_{1}^{G B}=\frac{T S_{1} \times G_{F}}{100}$

$T_{n}^{G B}=\frac{T S_{n} \times G_{F}}{100}$

where $G_{F}$ is the guard band factor and it is assumed based on the average drift period. On the other hand, before

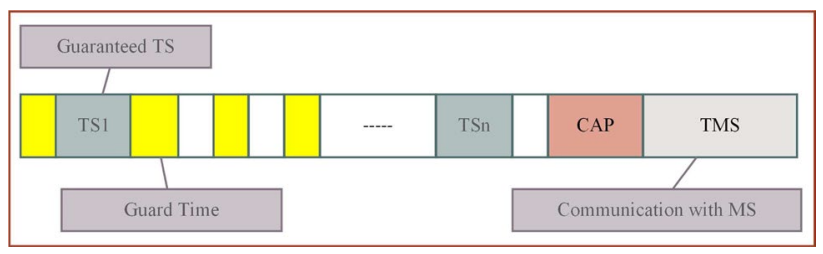

Fig. 3 Allocation of time slots for all sensor nodes with guard band times 
every TS, $T_{G B}$ is placed as shown in Fig. 3. Sensor nodes go into sleep mode on successful TS assignment and only woke up to relay information in assigned TSs to the sink. This method avoids passive attention during other sensor nodes are in active mode. This method of assignment minimizes the consumption of energy and provides stable operation of the network.

\subsection{Data transmission from the sink}

All sensor nodes communicate with the sink during TSs for collision-free transmission. Figure 4 shows the queuing model for the sink (personal server), which describes data transmission from the sink to the internet. This model classifies incoming traffic into critical and normal traffic according to the critical table [21]. The scheduler i.e, the sink assigns TSs to every traffic. Based on the path state information, the path selector allocates the optimized route for data transmission. The path state information is provided by the cost function defined in Eq. (13). Once a path is established, the information is transmitted to the internet via the gateway. From the internet, the information can be monitored by doctors and families. The data transmission stage operation has been shown in Fig. 4.

Cost Function $=\frac{1}{(\operatorname{distance}(S(i))}$

Finally, the sink captures and analyzes the input from every sensor, and it also communicates with the loT. The succeeding iteration begins immediately after the broadcast of data, and this entire cycle is performed in the same manner.

The following pseudo code explains the route selection according to the priority of the sensor by considering the distance, residue energy, temperature, and the hop count within that route. The notation used for the pseudocode is presented in Table 2.

Table 2 List of notations

\begin{tabular}{ll}
\hline Parameter & Value \\
\hline $\mathrm{n}$ & Number of sensor nodes \\
$\mathrm{i}$ & Varies from 1 to $\mathrm{n}$ \\
$\mathrm{dr} \_\mathrm{i}$ & distance for route $\mathrm{i}$ \\
$\mathrm{dr} \_\mathrm{i}+1$ & distance for route $\mathrm{i}+1$ \\
$\mathrm{Tr} \_\mathrm{i}$ & The temperature at route $\mathrm{i}$ \\
$\mathrm{Tr}_{-} \mathrm{i}+1$ & The temperature at route $\mathrm{i}+1$ \\
$\mathrm{HCr} r_{-} \mathrm{i}$ & Hop count of route $\mathrm{i}$ \\
$\mathrm{HCr} \mathrm{i}+1$ & Hop count of route $\mathrm{i}+1$ \\
\hline
\end{tabular}

\section{Data Transmission from the sink}

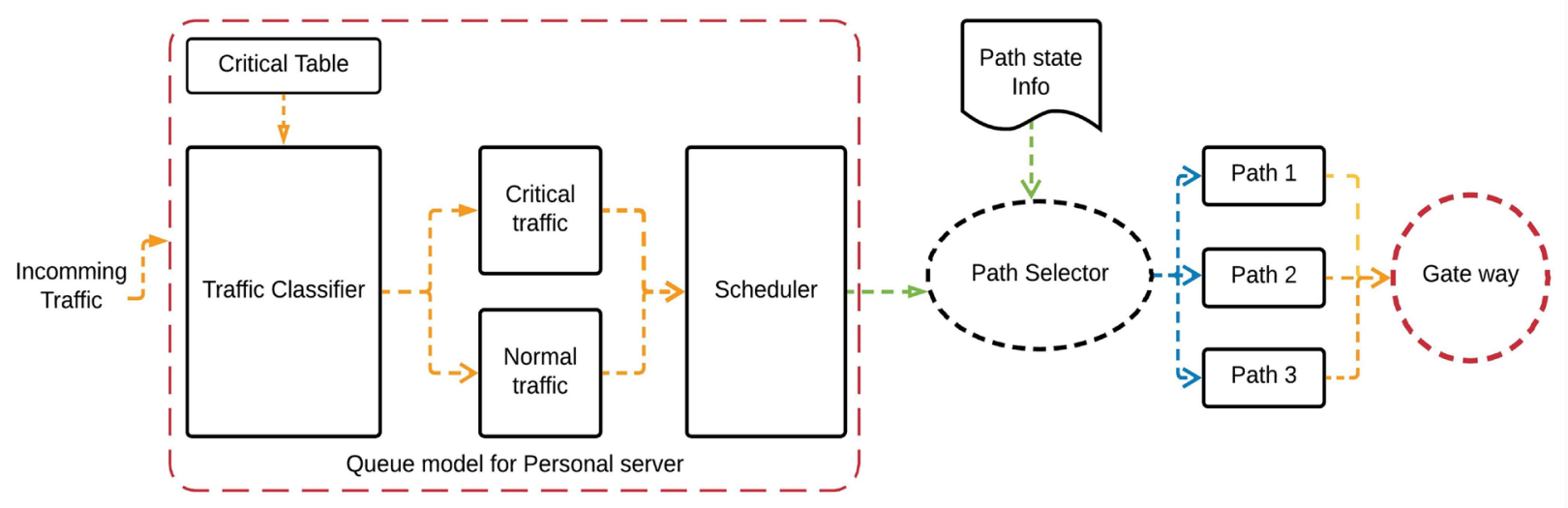

Fig. 4 Data transmission from the sink 


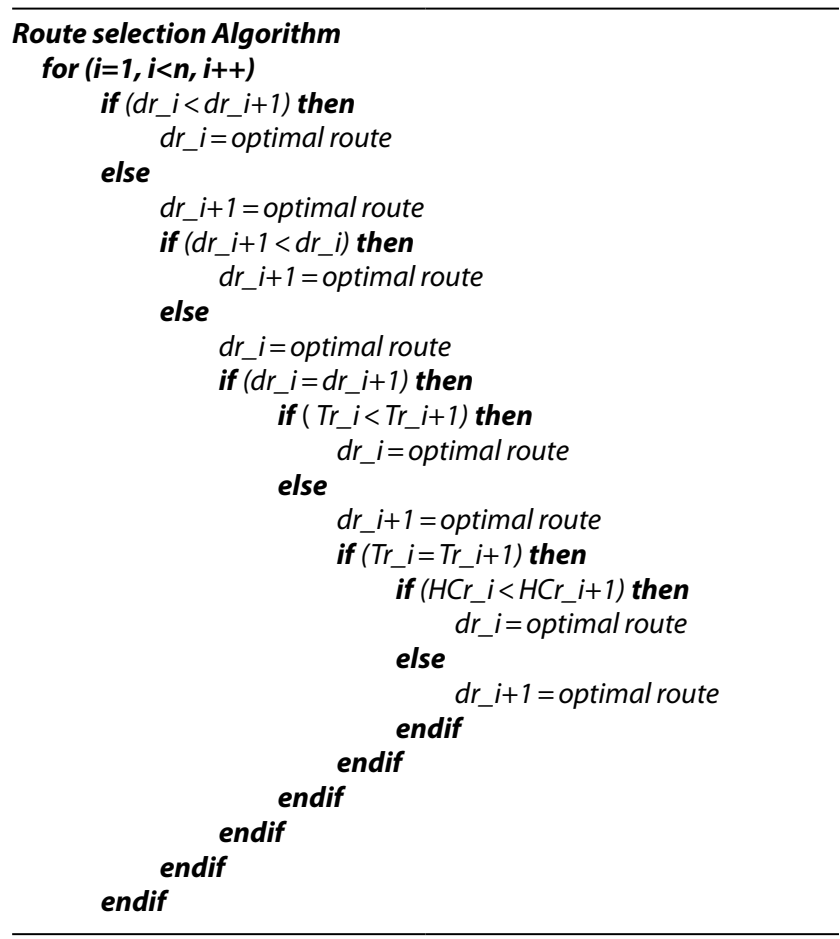

\section{Results}

The performance of the hybrid protocol is validated by comparing with conventional protocols such as Stable Increased throughput, Multihop Link Efficient protocol (SIMPLE) [18], Adaptive Threshold-based Thermal-aware Energy-efficient Multihop Protocol (ATTEMPT) [17], and Reliable Energy-Aware Stable Topology (REAST) [16] protocols with two ULP transceivers nRF2401A and nRF24L01. The network area considered is $0.8 \times 1.8 \mathrm{~m}^{2}, 8$ sensor nodes are placed at diverse places with an initial energy of 0.4 Joules. At the center of the patient's body, the sink is deployed. Table 3 presents the wearable sensors, their specific characteristics. The simulation parameters that are taken from Nordic ULP nRF2401A and nRF24L01 transceivers are presented in Table 4. The simulations are carried out for the entire network by considering the collision-free

Table 3 Wearable sensors and their parameters.

\begin{tabular}{llllll}
\hline Parameter & \multicolumn{5}{l}{ Warble sensors } \\
\cline { 2 - 6 } & BP & TEMP & EMG & EEG & ECG \\
\hline QoS & Yes & Yes & Yes & Yes & Yes \\
Data type $(\mathrm{Kb} / \mathrm{s})$ & 0.016 & 0.12 & 300 & 43.2 & 288 \\
Accuracy & 8 & 8 & 16 & 12 & 8 \\
Power consumption & High & Low & Low & Low & Low \\
Privacy & High & Low & High & High & High \\
Bandwidth $(\mathrm{Hz})$ & $0-150$ & $0-1$ & $0-10 \mathrm{~K}$ & $0-1$ & $100-1 \mathrm{~K}$ \\
\hline
\end{tabular}

Table 4 Simulation parameters considered for validation of proposed protocol

\begin{tabular}{lll}
\hline Parameter & \multicolumn{2}{l}{ Transceiver } \\
\cline { 2 - 3 } & nRF2401A & nRF24L01 \\
\hline Tx Current (mA) & 17.4 & 10.5 \\
Rx Current (mA) & 19.7 & 18 \\
Voltage (V) & 2.1 & 1.9 \\
$E_{\text {tx-ele }}$ & 96.9 & 16.7 \\
E $_{\text {rx-ele }}$ & 172.8 & 36.1 \\
Eamp & 2.71 & 1.97 \\
\hline
\end{tabular}

channel for $10^{4}$ rounds using MATLAB. Four network parameters such as stability period, data transmission rate, residue energy, and path loss are taken into account during a simulation.

\subsection{Analysis of stability periods}

The stability period is the measure of the network lifetime, and it is defined as the first node of failure after the establishment of the network, which is measured in terms of rounds. According to ATTEMPT protocol [20], the biosensor nodes are not in line with the corresponding energy levels. Therefore, there is an impact on the stability period restricted to 2100 rounds associated with nRF2401A. By simulating the same protocol with nRF24L01, the stability increased by 1200 rounds. In SIMPLE [19], the nodes which are transmitting high data are quickly depleted than low data rates due to unequal distribution of energy; however, a limited period of stability for 3500 and 4300 rounds in nRF2401A and nRF24L01 transceivers respectively. However, REAST [21] and hybrid protocols achieve outstanding performance, i.e, stability period is up to 9000 rounds. This improvement of nRF2401A and nRF24L01 transceivers resulting in hybrid transmission and synchronization is given in Fig. 5.

\subsection{Analysis of data transmission rate}

The proposed protocol's data transmission rate is specified based on the number of packets broadcast from the sink to the gateway. Figure 6 shows the data transmission rate of the proposed protocol is of the order of $6 \times 10^{4} \mathrm{bits} / \mathrm{sec}$ with nRF2401A and $9 \times 10^{4}$ bits/sec with nRF24L01.

The data rate of the proposed protocol is better than conventional protocols. The improvement is based on its optimized energy efficiency as specified from Eqs. (3) to (6) and cost function mentioned in Eq. (10). Due to the increased energy efficiency and a lifetime of the network, the data transmission rate of the entire network is increased. As compared with nRF2401A, the data 

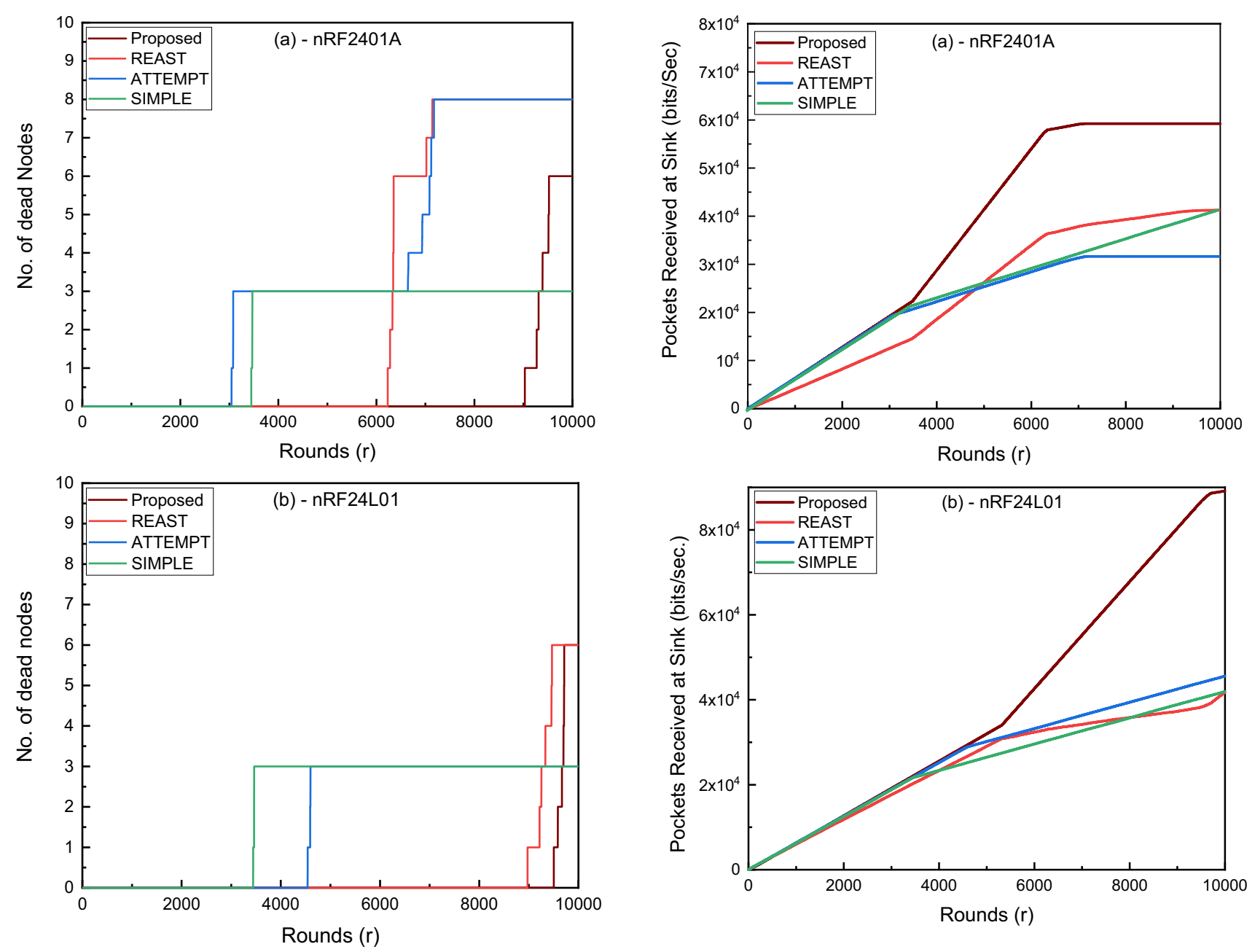

Fig. 5 Comparison of stability period of proposed protocol with SIMPLE [19], ATTEMPT [20], REAST [21] using ULP transceivers (a) nRF2401A and (b) nRF24L01

transmission rate of nRF24L01 is improved by $30 \%$ because of its low power operation as shown in Table 2. The graphical representation of the data transmission rate at the sink for SIMPLE, ATTEMPT, REAST, and proposed protocol with nRF2401A and nRF24L01 transceivers are shown in Fig. 7.

\subsection{Analysis of residual energy}

The residual energy of REAST [21] and the proposed protocol are superior when compared with SIMPLE [19] and ATTEMPT [20] with nRF2401A transceiver. In the case of nRF24L01, the SIMPLE and ATTEMPT protocols improved their performance at the cost of the data rates. However, the REAST and proposed protocols still have a good response, without compromising the data rates. This improvement is achieved by avoiding inactive attending and surplus hearing of sensors, which minimizes energy consumption due to periodic sleep and wake-up. The

Fig. 6 Comparison of data transmission rates of proposed protocol with SIMPLE [19], ATTEMPT [20], REAST [21] using ULP transceivers (a) nRF2401A and (b) nRF24L01

residual energy of proposed and REAST [21] protocols is doubled as the comparison with ATTEMPT [20] and SIMPLE [19] protocols with nRF2401A and it is depicted in Fig. 8.

\subsection{Analysis of path loss}

The human body path loss coefficient is given in Eqs. (8) and (9), which is a function of distance, and the operating frequency. The operating frequency $(f)$ is $2.4 \mathrm{GHz}$ and distance $(d)$ is the transmission distance from the sink node to the corresponding sensor node [21, 22].

$$
\begin{aligned}
& P L=\sigma_{S}+\left[n \times 10 \log _{10}\left(\frac{d}{d_{0}}\right)\right]+P L_{0} \\
& P L_{0}=n \times 10 \log _{10}\left(\frac{4 \pi f d}{c}\right)^{2}
\end{aligned}
$$



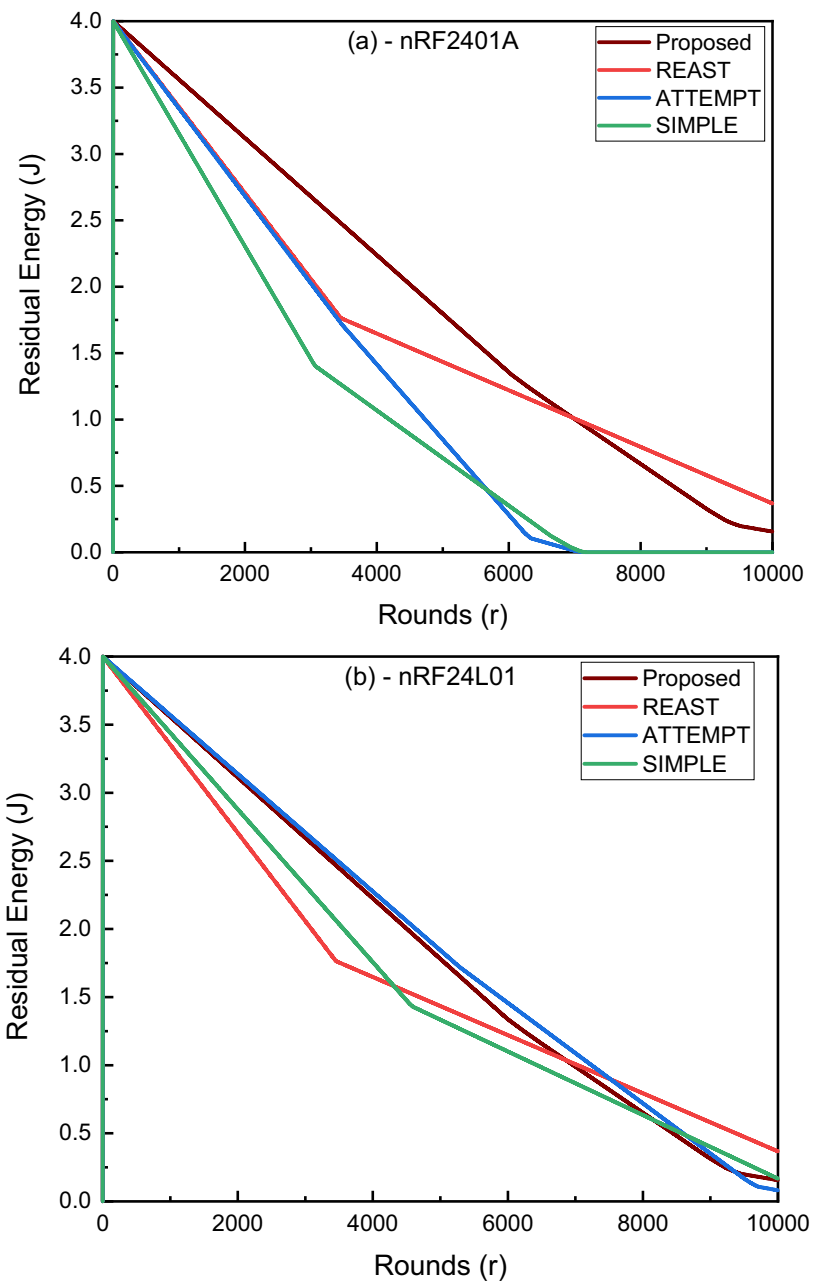

Fig. 7 The complete network comparison of the proposed protocol with SIMPLE [18], ATTEMPT [17], REAST [16] using ULP transceivers nRF2401A and nRF24L01 by considering (a) Stability period, (b) Data transmission rate, (c) Residual energy, and (d) Path loss

where $f=$ frequency of propagating wave, $c=$ speed of light, $\sigma_{S}=$ co-efficient path loss of the human body (3.38 to 4.1 )

The comparison of the proposed protocol with ATTEMPT [21], SIMPLE [19], and REAST [21]. The proposed protocol optimizes the $d$ is by a multihope transmission which in turn minimizes the path loss and is shown in Fig. 9.

\section{Discussion}

The overall network performance with ULP transceivers nRF2401A and nRF24L01 is shown in Fig. 7. Figure 7a shows a comparison of stability periods of conventional protocols with the proposed protocol. The REAST [21] protocol with nRF24L01 transceiver is having a maximum
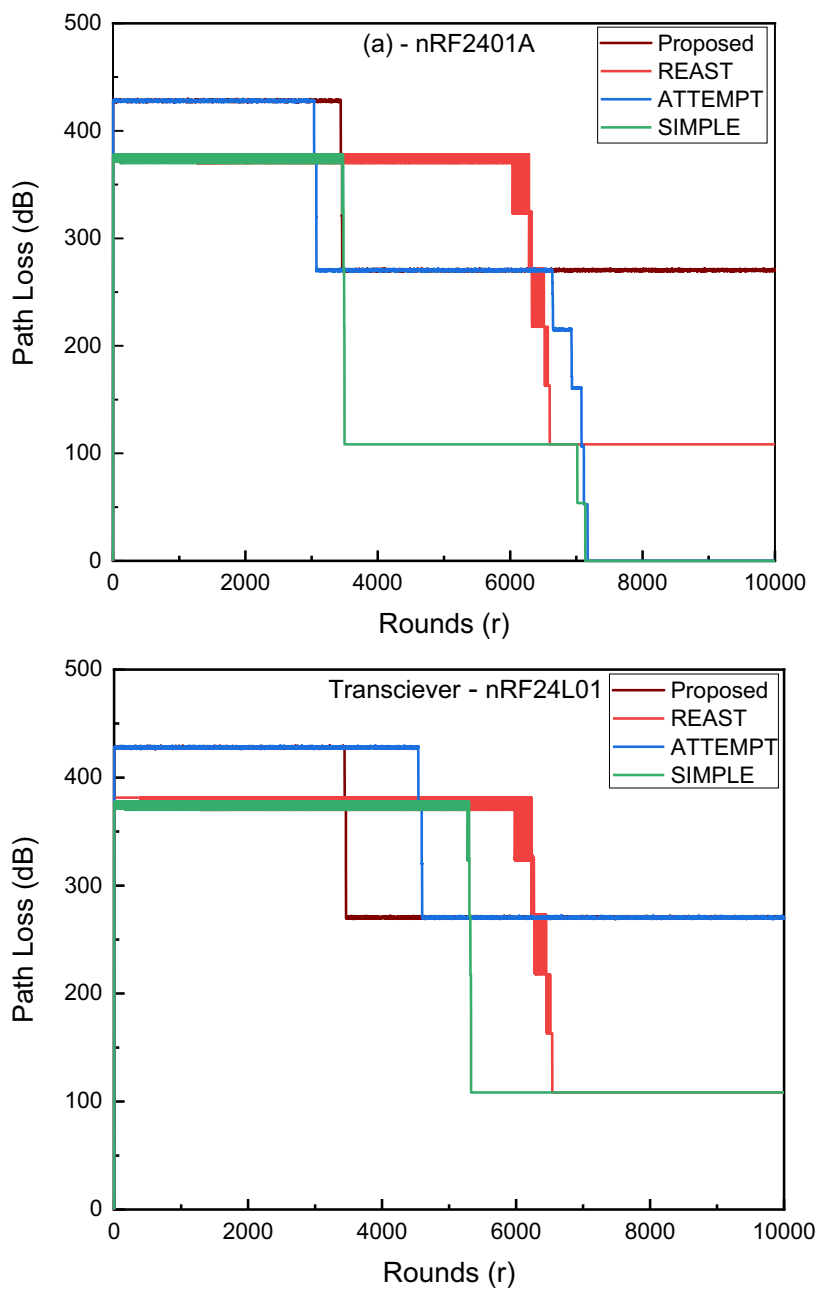

Fig. 8 Comparison of residual energy of proposed protocol with SIMPLE [19], ATTEMPT [20], REAST [21] using ULP transceivers (a) nRF2401A and (b) nRF24L01

stability period of 9200 rounds as a comparison with all other protocols. However, the proposed protocol provides the same stability period for both transceivers and its stability period is approximately equal to that of REAST [21] protocol.

Figure $7 \mathrm{~b}$ shows the comparison of the data transmission rate of conventional protocols with the proposed protocol. The proposed protocol provides a better data transmission rate as compared with the remaining protocols by using both transceivers. The proposed protocol having more than 30\% better performance with nRF24L01 transceiver. Figure 7c shows the comparison of the residual energy of conventional protocols with the proposed protocol. The ATTEMPT [20] protocol with nRF24L01 transceiver is having maximum residual energy of 1.5 Joules as a comparison with all other protocols. However, the proposed protocol provides the same stability period for both transceivers, 
(a)

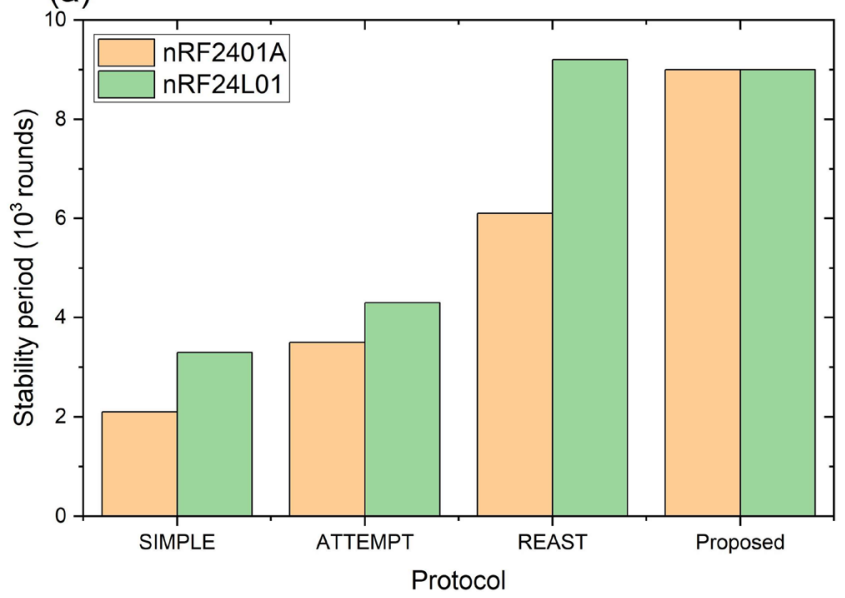

(c)

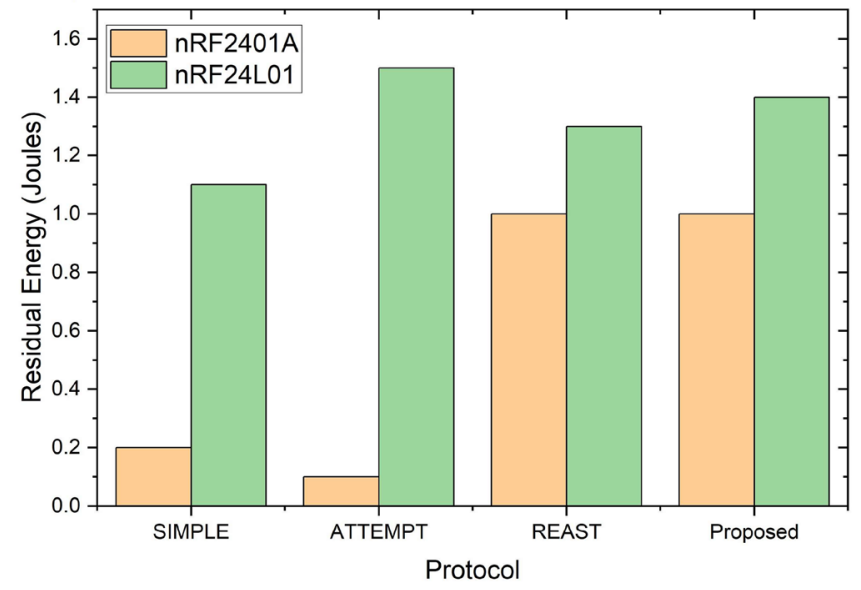

(b)

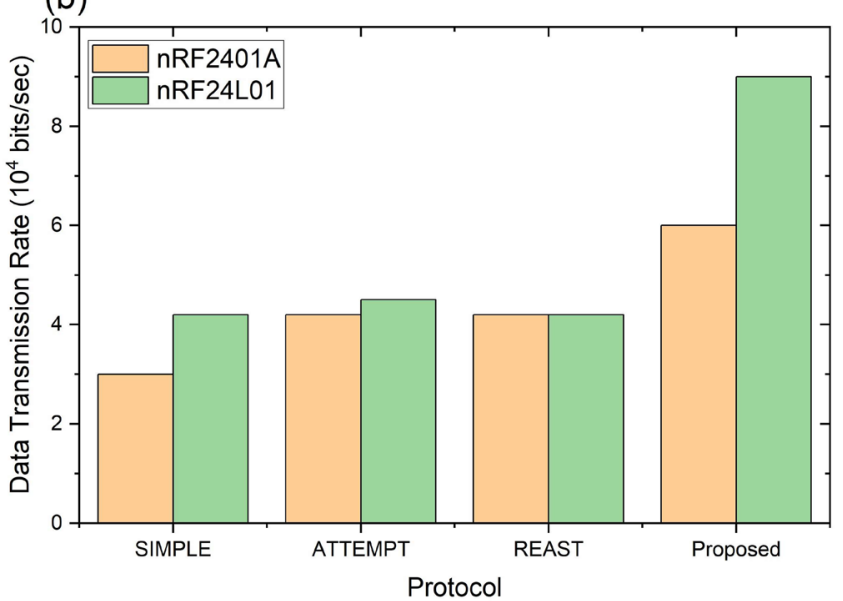

(d)

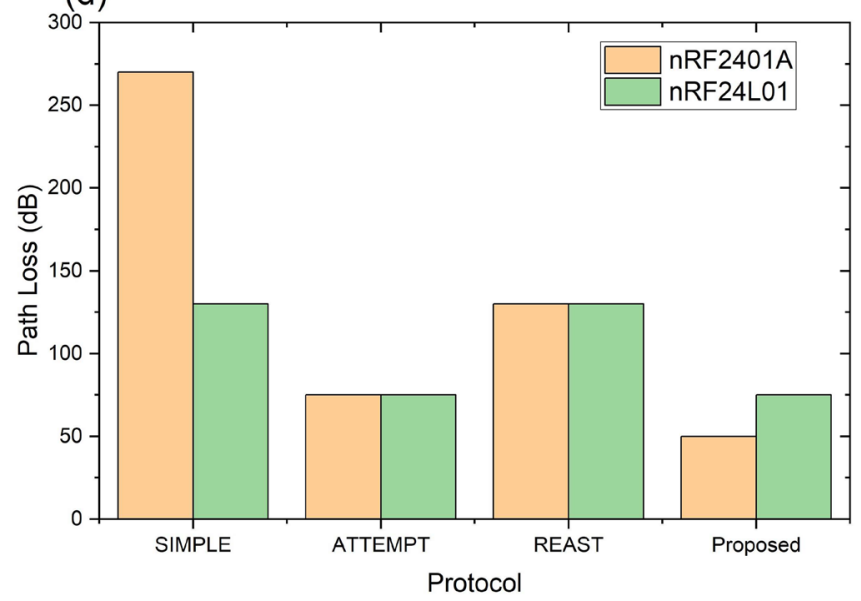

Fig. 9 Comparison of path loss of proposed protocol with SIMPLE [19], ATTEMPT [20], REAST [21] using ULP transceivers (a) nRF2401A and (b) nRF24L01

and its residual energy is approximately equal to that of ATTEMPT [20] protocol. The nRF24L01 provides fairly good performance in all the protocols, because of its low power operation. The comparison of path loss between conventional protocol and proposed protocol is shown in Fig. 7d. ATTEMPT [20] and the proposed protocol provide less path loss for both the transceivers.
The proposed protocol performance with nRF2401A transceiver is improved by $25 \%$ in contrast with conventional protocols is illustrated in Table 5. Similarly, with nRF24L01, the performance is improved by nearly $35 \%$ as compared with SIMPLE and ATTEMPT protocols. Besides, the nRF24L01 has better as compared with nRF2401A. Because of its low power consumption, it prevents damage
Table 5 Overall improvement of the proposed protocol with conventional protocols using nRF2401A

\begin{tabular}{lllll}
\hline Parameter/Protocol & $\begin{array}{l}\text { Stability period } \\
\text { (Rounds) }\end{array}$ & $\begin{array}{l}\text { Data transmission } \\
\text { rate }(\mathrm{Kb} / \mathrm{s})\end{array}$ & $\begin{array}{l}\text { Residual energy } \\
\text { (Joules) }\end{array}$ & Path loss (dB) \\
\hline SIMPLE & 3200 & 30 & 0.2 & 270 \\
ATTEMPT & 4300 & 40 & 0.1 & 75 \\
REAST & 6100 & 40 & 1.0 & 125 \\
PROPOSED & 9000 & 50 & 1.0 & 50 \\
Proposed improvement (\%) & 42.85 & 25 & 0 & 33.33 \\
Overall improvement (\%) & (Sum of individual parameter performance/number of param- \\
& eters) $=(100.87 / 4)=\mathbf{2 5}$ &
\end{tabular}


to patient-sensitive organs resulted from the radiation of the sensor nodes. Finally, form the above results the proposed protocol is providing far better performance with the nRF24L01 transceiver; hence it is more suitable for e-Healthcare systems.

\section{Conclusion}

This paper proposed a hybrid routing protocol to accomplish energy efficiency in WBANs to support e-Health care systems. The proposed protocol utilizes hybrid communication between the sink and the sensor nodes to enhance the network lifetime of the overall network by optimizing energy consumption. Furthermore, the protocol utilizes a novel synchronization scheme with a variable TSs assignment to minimize the energy consumption of the network. The network is simulated at $10^{4}$ rounds with ULP radio transceivers nRF2401 and nRF24L01 using four network parameters. The proposed protocol achieves $25 \%$ better performance with nRF $2401 \mathrm{~A}$ and $35 \%$ better performance with the nRF24L01 in contrast with the conventional protocols. The proposed protocol avoids damage to patients' sensitive organs due to radiation losses resulting from temperature effects at the sensor node. Hence, the proposed protocol with Nordic ULP nRF24L01 is most suitable for eHealth care systems.

Acknowledgments We thank Koneru Lakshmaiah Education Foundation (KLEF), Green Fields, Vaddeswaram, Andhra Pradesh, and SR University, Warangal for supporting us during this work.

Complaince with ethical standards

Conflict of interest The authors declare that they have no conflict of interest.

\section{References}

1. Alwan A (2011) Global status report on noncommunicable diseases 2010. WHO, Geneva, Switzerland. Available online. http://www.who.int/nmh/publications/ncd_report_full_en.pdf. Accessed 1 May 2016

2. Hopman P, Heins MJ et al (2015) Health care utilization of patients with multiple chronic diseases in The Netherlands: differences and underlying factors. Eur J Intern Med 26(3):190-196

3. Prasad CR, Bojja P (2019) Im-reast: an improved reliable, energy aware and stable topology for wireless body biosensor networks in health-care systems. ARPN J Eng Appl Sci 14(10):1971-1978

4. Liao Y, Leeson MS, Higgins MD (2016) A communication link analysis based on biological implant wireless body area networks. Appl Comput Electromagn Soc J 31:619-628

5. Movassaghi S, Abolhasan M et al (2014) Wireless body area networks: a survey. IEEE Comm Surv Tut 16(3):1658-1686
6. Rajendra Prasad C, Bojja P (2019) Reliable energy aware MAC protocol for wireless body bio-sensor networks. IJITEE 8(8):2604-2608

7. Behera TM, Samal UC, Mohapatra SK (2018) Energy-efficient modified LEACH protocol for IoT application. IET WSS 8(5):223-228

8. Liao Y, Leeson MS et al (2016) Analysis of in-to-out wireless body area network systems: towards QoS-aware health loT appl. Electronics 5(3):38

9. Fan YJ et al (2014) loT-based smart rehabilitation system. IEEE Trans Industr Inform 10(2):1568-1577

10. Ullah S, Chen M, Kwak KS (2012) Throughput and delay analysis of IEEE 802.15. 6-based CSMA/CA protocol. J Med Syst 36(6):3875-3891

11. Rajendra Prasad C, Bojja P (2018) A survey on routing protocols in wireless body area networks for medical applications. JARDCS 10(10):92-97

12. Javaid N, Ahmad A, Nadeem Q, Imran M, Haider N (2015) iMSIMPLE: iMproved stable increased-throughput multi-hop link efficient routing protocol for wireless body area networks. Comput Hum Behav 51:1003-1011

13. Smail O, Kerrar A, Zetili Y, Cousin B (2016) ESR: Energy aware and Stable Routing protocol for WBAN networks. In: 2016 international wireless communications and mobile computing conference (IWCMC), September, IEEE, pp 452-457

14. Chen C, Knoll A, Wichmann HE, Horsch A (2013) A review of three-layer wireless body sensor network systems in healthcare for continuous monitoring. MIOT 2(3):24-34

15. Tseng HW, Wu RY, Wu YZ (2016) An efficient cross-layer reliable retransmission scheme for the human body shadowing in ieee 802.15. 6-based wireless body area networks. IEEE Sensors J 16(9):3282-3292

16. Sayrafian-Pour K, Yang WB, Hagedorn J, Terrill J, Yazdandoost KY (2009) A statistical path loss model for medical implant communication channels. In: 2009 IEEE 20th international symposium on personal, indoor and mobile radio communications, September, IEEE, pp 2995-2999

17. Reusens $E$, Joseph W, Latré B, Braem B, Vermeeren G, Tanghe $E$ et al (2009) Characterization of on-body communication channel and energy efficient topology design for wireless body area networks. IEEE Trans Inf Technol Biomed 13(6):933-945

18. Hassan MN, Murphy L, Stewart R (2016) Traffic differentiation and dynamic duty cycle adaptation in IEEE 802.15. 4 beacon enabled WSN for real-time applications. Telecommun Syst 62(2):303-317

19. Nadeem Q, Javaid N, Mohammad SN, Khan MY, Sarfrazm S, Gull M (2013) SIMPLE: stable increased-throughput multi-hop protocol for link efficiency in wireless body area networks, BWCCA. In: 2013 eighth international conference on, vol 221(226), pp. 28-30. https://doi.org/10.1109/BWCCA.2013.42.

20. Ahmad A, Javaid $\mathrm{N}$ et al (2014) RE-ATTEMPT: a new energyefficient routing protocol for WBSNs. Int J Distrib Sens Netw 10(4):464010

21. Prasad CR, Bojja P (2019) A reliable, energy aware and stable topology for bio-sensors in health-care applications. J Commun 14(5):390-395. https://doi.org/10.12720/jcm.14.5.390-395

22. Janapati R, Balaswamy C, Soundararajan K (2018) Enhancement of localized routing using CDPSO in WSN. In: 2018 conference on signal processing and communication engineering systems (SPACES), January, IEEE, pp 16-19

Publisher's Note Springer Nature remains neutral with regard to jurisdictional claims in published maps and institutional affiliations. 